
ДВИЖЕНИЯ ВАГОНА НА УЧАСТКАХ ТОРМОЗНЫХ ПОЗИЦИЙ СОРТИРОВОЧНОЙ ГОРКИ

\author{
Туранов Х.Т. ${ }^{1}$, Саидивалиев Ш.У. ${ }^{2}$ \\ ${ }^{1}$ Уральский государственный университет путей сообщения, г. Екатеринбург, Россия \\ 2 Ташкентский институт инженеров железнодорожного транспорта, г. Ташкент, Узбекистан
}

\title{
Аннотация
}

Существующие методики расчёта сортировочных горок направлены, в основном, на определение высоты сортировочной горки, а кинематические параметры движения вагона в зоне затормаживания не принимаются во внимание. В статье представлены расчётные формулы и методика определения пути торможения вагона на участках тормозных позиций сортировочной горки. Разработанная методика основана на теореме теоретической механики об изменении кинетической энергии для несвободной материальной точки в конечной форме. Показано, что путь прохождения вагоном участка торможения можно определить по формуле пути, при известной величине ускорения при затормаживании вагона. С использованием разработанных формул выполнен расчёт величины тормозного пути, в зависимости от времени торможения и от скорости движения отцепа. Относительная погрешность расчёта при одном и том же значении начальной скорости не превысила $3.7 \%$, что подтверждает корректность разработанных расчётных формул. Предлагаемая методика позволяет при известной величине пройдённого вагоном по участку тормозных позиций расстояния определять время, в течение которого произойдёт равноускоренное движение вагона на данном участке сортировочной горки. Результаты выполненных исследований могут быть использованы для корректного решения задач по расчёту и проектированию сортировочных горок.

Ключевые слова: сортировочная горка, тормозные позиции, вагон, кинетическая энергия, количество движения, кинематические параметры, принцип Даламбера, теорема об изменении кинетической энергии, теоремы о движении центра инерции системы материальных точек, теоремы об изменении количества движения.

\section{1. Введение*}

Отличительной особенностью железнодорожного транспорта является возможность обеспечения безопасного и плавного движения подвижного состава на прямых участках железнодорожного пути и в кривых. Для этого железнодорожный подвижной состав должен обладать ходовой стабильностью и необходимыми поворотными характеристиками [1].

В настоящее время используются различные современные научные методы для исследования сложной проблемы динамики железнодорожных вагонов. Актуальность решения данной проблемы связана с требованиями по увеличению скорости и грузоподъёмности, стремление к соблюдению которых вызывает возникновение новых задач, в частности, задач снижения износа и обеспечения устойчивости подвижного состава. Решение этих задач требует системного подхода к проблеме на основе использования фундаментальных теорий и положений [2-4].

Например, в работе [5] исследуется взаимодействие подвижного состава и железнодорожного пути на длинном и наклонном участке скоростной железной дороги в условиях торможения. В разработанной авторами модели транспортное средство рассматривается как многожёсткая система с 21-й степенью свободы. Железнодорожный путь моделируется в виде балки
Эйлера. Для уменьшения степени свободы модели авторами предложен метод «кругового пути».

Особенностью разработанной модели динамического взаимодействия вагона и железнодорожного пути является комбинация двух моделей - модели продольного взаимодействия колеса и рельса, основанной на теории ползучести Полаха, применяемой для описания состояния большой ползучести в результате сильного торможения [6], и модели, использующей положения продольной теории жёсткого контакта. Результаты расчёта динамических характеристик подструктур исследуемой системы для условий торможения транспортного средства, полученные с использованием данных двух моделей, различаются незначительно. Однако модель ползучести Полаха способна учитывать ползучесть в системе колесо-рельс в процессе торможения и позволяет определять критические значения параметров этой системы. Результаты расчётов на данной модели для различных условий показывают, что увеличение тормозного момента является причиной скольжения некоторых или всех колёс вагона, что приводит к нарушению контакта колесорельс. Поэтому тормозной момент должен быть меньше определённого значения для того, чтобы ограничить тормозной путь и время торможения и предотвратить скольжение колеса по рельсу. Согласно расчё-

( Туранов Х.Т.1, Саидивалиев Ш.У., 2019. 
там, приведённым в настоящей статье, величина тормозного момента для условий сухого и мокрого пути должна составлять, соответственно, 7 и 4 кН·м.

Динамика заторможенного железнодорожного вагона на участках тормозных позиций исследовалась в работах $[7,8]$. Важной задачей является регулирование скоростей в зонах торможения вагона на участках тормозных позиций. Сортировочные горки большой мощности оборудуются вагонными замедлителями различных типов, которые являются основными тормозными средствами для регулирования скоростей скольжения вагонов [9]. При этом осуществляется торможение двух видов - интервальное и прицельное (целевое). Интервальное торможение обеспечивает необходимые интервалы между вагонами для безопасного прохождения их по стрелочным переводам и тормозным устройствам в пределах спускной части. Прицельное (целевое) торможение позволяет регулировать скорость движения вагона в зависимости от расстояния, которое он должен пройти на подгорочном парке. В работе [10] описаны разнообразные факторы (ходовые свойства вагонов, дальность пробега, наличие кривых и стрелок по маршруту следования вагона по профилю горки, погодные условия, а также человеческий фактор), влияющие на процесс торможения вагонов на сортировочных горках, а также определяющие применение каждой из тормозных позиций (первой, второй, третьей - 1ТП, 2ТП, 3ТП).

Задача расчёта времени движения и пути торможения вагона на участках тормозных позиций считалась трудноразрешимой $[11,12]$. В статьях $[13,14]$, как и в существующей методике горочных конструктивных и технологических расчётов, эта задача решена с использованием понятия «мощности тормозных позиций». Заметим, что в работах $[13,14]$ при выполнении горочных расчётов, как на скоростных, так и на тормозных участках, не используются формулы для определения ускорения движения вагона.

Инженерная проблема нахождения времени затормаживания и пути прохождения вагона на участках тормозных позиций недостаточно исследована.

Целями настоящего исследования являются:

- построение математических моделей движения вагона на основе использования классических положений теоретической механики и вывод соотношений для определения пути затормаживания вагона;

- выполнение расчётов с использованием разработанных математических моделей для оценки их корректности и применимости для выполнения горочных расчётов применительно к зонам торможения вагона на второй и третьей тормозных позициях.

\section{2. Методы}

В настоящем исследовании предлагается решение инженерной задачи определения кинематических характеристик вагона четырьмя способами на основе:

1. основного закона динамики для неидеальных связей (принципа Даламбера);

2. теоремы о движении центра инерции системы материальных точек;

3. теоремы об изменении кинетической энергии для несвободной материальной точки в конечной форме;

4. теоремы об изменении количества движения точки.

Запишем теорему об изменении кинетической энергии для несвободной материальной точки на отрезке $A B$ [11], где возможно движение вагона, с учётом начальной скорости $v_{0}$ применительно к рассматриваемой задаче в виде

$$
\frac{G}{2 g}\left(v_{B}^{2}-v_{A}^{2}\right)=A_{F x}, \kappa \mathrm{H},
$$

где $v_{A}, v_{B}$ - скорость движения вагона, соответственно, в точке $A$ (начало движения) и $B$ (конец движения); $G$ - сила тяжести, кН; $\mathrm{g}$ - ускорение свободного падения, $\mathrm{M} / \mathrm{c}^{2}$,

$$
A_{F x}=A_{G x}+A_{F f}, \mathrm{\kappa H},
$$

где $A_{G x}$ - работа силы тяжести $G x$ (через её проекцию на ось $x$ ) по перемещению на расстояние $x_{A B}$ (между точками $A$ и $B$ ), кН; $A_{F f}$ - работа силы трения $F f$ (в общем случае - различных сил сопротивления $F r$ ) по перемещению на расстояние $x_{A B}$, кН [11].

Величины $A_{G x}$ и $A_{F f}$ определяются по следующим формулам

$$
\begin{aligned}
& A_{G x}=G_{x} x_{A B}=G \sin \psi x_{A B}, \\
& A_{F f}=-F_{f} x_{A B}=-k_{f} G \cos \psi x_{A B},
\end{aligned}
$$

где $k_{f}=0.25$ - коэффициент трения колёс железнодорожного вагона о рельсовые нити [14], $\psi$ - угол наклона участка $A B$ профиля горки.

Приращение кинетической энергии системы $\Delta E$ равно сумме соответствующих работ активных сил $A_{G x}$ и реакций связей $A_{F f}$, то есть

$$
\Delta E=A_{G x}+A_{F f}, \text { кН. }
$$

Подставляя выражения (3) и (4) в (2), с учётом (1) и после упрощений, получаем следующую формулу расчёта скорости движения вагона в зоне торможения на участках тормозных позиций

$$
v_{B i}^{2}-v_{A i}^{2}=2 g\left(\sin \psi_{i}-k_{f} \cos \psi_{i}\right) x_{A B i},
$$

где $i$ - номера участков профиля пути $(i=1, \ldots 9) ; v_{A i}$, $v_{B i}-$ скорости движения, соответственно, в точках $A i$ (начало движения на $i$-м участке профиля сортировочной горки) и $B i$ (конец движения на $i$-м участке); $\psi_{i}-$ угол наклона участка $A i B i$ профиля горки.

При $x_{A B i}=l_{i}$, где $l_{i}-$ длина тормозного пути на $i$-м участке профиля, м, выражение (6) перепишется следующим образом

$$
v_{B i}^{2}=v_{A i}^{2}+2 g\left(\sin \psi_{i}-k_{f} \cos \psi_{i}\right) l_{i} .
$$

При $v_{B i}=0$ получаем

$$
0=v_{A i}^{2}+2 g\left(\sin \psi_{i}-k_{f} \cos \psi_{i}\right) l_{i}
$$

Из выражения (8) получаем формулу расчёта длины тормозного пути $l i$ 


$$
l_{i}=\frac{v_{A i}^{2}}{2 g\left(k_{f} \cos \psi_{i}-\sin \psi_{i}\right)}, \mathrm{\text {. }}
$$

Учитывая, что для малых углов (менее $5^{\circ}$ ), которые имеют различные элементы профиля сортировочной горки, $\sin \psi_{i} \approx \psi_{i}=i_{i}\left(i_{i}-\right.$ уклон $i$-го участка в \%о) и $\cos \psi_{i}$ $\approx 1$, то формулы (7) и (9) примут вид

$$
\begin{aligned}
& v_{B i}^{2}=v_{A i}^{2}+2 g\left(i_{i}-k_{f}\right) l_{i}, \\
& l_{i}=\frac{v_{A i}^{2}}{2 g\left(k_{f}-i_{i}\right)} .
\end{aligned}
$$

Как видно из выражения (11), величина тормозного пути $l_{i}$ прямо пропорциональна квадрату начальной скорости $v_{A i}$ и обратно пропорциональна коэффициенту трения $k_{f}$, а также наклону участка пути $i_{i}$.

Абсолютное значение ускорения вагона при равнозамедленном движении в зоне торможения $\left|a_{i}\right|$ определяется по формуле [11]

$$
\left|a_{i}\right|=\frac{\left|\Delta F_{f i}\right|}{M_{r 0}} 10^{3}, \mathrm{M} / \mathrm{c}^{2},
$$

где $\left|\Delta F_{f i}\right|$ - результирующая сила, под воздействием которой колёсные пары вагона вынуждены скользить по поверхностям катания рельсовых нитей и тормозным шинам вагонного замедлителя в зоне торможения на участках тормозных позиций [11], кН; $M_{r 0}-$ приведённая масса вагона с грузом при чистом скольжении колёсных пар, принуждённо «зажатых» тормозными шинами вагонного замедлителя в зоне торможения на участках тормозных позиций и рассматриваемых как пара сухого трения «сталь по стали».

Величина $\left|\Delta F_{f i}\right|$ определяется по формуле

$$
\left|\Delta F_{f i}\right|=F_{x i}+\left|F_{c i}\right| \text {, }
$$

где $F_{x i}-$ сила, под воздействием которой вагон входит в зону торможения на участках тормозных позиций с учётом воздействия проекций силы попутного ветра малой величины, кН; $F_{c i}$ - сила сопротивления, под воздействием которой вагон может быть заторможен вплоть до его остановки вагонным замедлителем - сопротивление сухому трению скольжения контактирующих поверхностей обода колёсных пар и тормозных шин вагонного замедлителя, основное (или ходовое) сопротивление, сопротивление от воздушной среды и ветра, сопротивление из-за снега или инея, кН.

Если известно значение ускорения движения согласно формуле (13) при равнозамедленном движении вагона $\left|a_{i}\right|$, то скорость движения до момента остановки вагона определяется по формуле скорости

$$
v_{f i}^{2}=v_{A i}^{2}+2\left|a_{i}\right| l_{i} .
$$

С использованием формулы скорости вида

$$
v_{f i}=v_{A i}+\left|a_{i}\right| t_{i},
$$

время торможения $t_{i}$ до момента остановки вагона $\left(t_{i}<t\right.$, где $t$ - текущее время, с) определится по формуле

$$
t_{i}=\frac{v_{A i}-v_{f i}}{\left|a_{i}\right|}, \mathrm{c} .
$$

Таким образом, применение теоремы об изменении кинетической энергии материальной точки в конечном виде в зонах торможения вагона на участках тормозных позиций с использованием выражений (9) или (11) позволяет рассчитывать длину пути прохождения вагоном участка торможения $l_{i}$.

Для вычисления $l_{i}$ рассматриваются следующие варианты, которые необходимы для задания начальной скорости входа вагона в зону торможения $v_{A i}=3.57 \mathrm{M} / \mathrm{c}$ :

- непосредственный вход на участок тормозной позиции первой колёсной пары $l_{A i}$ и/или колёсных пар передней тележки $l_{s t}$;

- вход вагона на участок на длину базы вагона.

Путь торможения вагона определяется по формуле

$l_{i}=v_{A i} t_{i}+\frac{1}{2} g\left(\sin \psi_{i}-k_{f} \cos \psi_{i}\right) t_{i}^{2}, \mathrm{M}$

Ускорение вагона при равнозамедленном движении в зоне торможения $\left|a_{i}\right|$, в отличие от выражения (12) можно определить как [11]

$$
\left|a_{i}\right|=a_{f}\left(i_{f i}-\left|w_{i}\right|\right)
$$

где $a_{f}=$ const - линейное ускорение вагона при равнозамедленном движении в зонах торможения на участках тормозных позиций $[11], \mathrm{M} / \mathrm{c}^{2} ;\left|w_{i}\right|-$ удельное сопротивление движению в зонах торможения на участках тормозных позиций, $\mathrm{H} / \kappa \mathrm{H} ; i_{f i}-$ приведённая величина уклона профиля горки на участках тормозных позиций, рассчитанная с учётом воздействия силы попутного ветра $F_{w x}$ (через проекцию этой силы), \%о [11].

Величина $a_{f}$ определяется по формуле

$$
a_{f}=\frac{G}{M_{r 0}} 10^{3} .
$$

Величина $i_{f i}$ определяется по формуле

$$
i_{f i}=i_{f x i}+k_{w x}
$$

где $k_{w x}$ - безразмерная величина, учитывающая ускоряющее воздействие силы попутного ветра $F_{w x}$ малой величины на вагон (через проекцию этой силы на ось $x$ ), принимаемая в долях от величины $G$. Если ветер отсутствует, то $k_{w x}=0$ [11].

В соответствии с формулой (18), выражение (17) примет вид

$$
l_{i}=v_{A i} t_{i}+\frac{1}{2} a_{f}\left(i_{f i}-\left|w_{i}\right|\right) t_{i}^{2}
$$

и

$$
l_{i}=v_{A i} t_{i}+\frac{1}{2} a_{f}\left|a_{i}\right| t_{i}^{2} .
$$

Момент остановки вагона $t_{i}$ определится как

$$
t_{i}=\frac{v_{A i}}{g\left(k_{f} \cos \psi_{i}-\sin \psi_{i}\right)} .
$$

Сравнивая формулу (17), полученную согласно теореме о движении центра инерции системы материальных точек, и формулу (9), выведенную на основе теоремы об изменении кинетической энергии материальной точки в конечном виде, с формулой пути, известной из курса элементарной физики (22), видно, что они имеют различия. 
Относительная ошибка расчёта пути торможения вагона, вычисленная по формулам (22) и (17), составила $1.52 \%$, а по формулам (22) и (21) - 9.2\%, что находится в пределах точности инженерных расчётов.

Путь торможения вагона, вычисленный по формуле (22) составляет $l_{i}=13.35 \approx 13.4 \mathrm{M}$, а по формуле (9) $l_{i}=12.86 \approx 12.9$ м. Относительная ошибка вычислений составила $3.7 \%$, что подтверждает корректность вывода формулы (9).

\section{3. Расчётный пример}

В качестве расчётного примера выбрана зона торможения на участке второй тормозной позиции сортировочной горки. Рассматривается случай входа вагона на участок на длину базы вагона $l_{B}$ (для платформы $-l_{B}$ $=9.72 \mathrm{м})$. При входе вагона на участок второй тормозной позиции $l_{B X 2}=1.0, \ldots, 2.0 \mathrm{м}$.

Исходные данные расчётного примера:

- сила тяжести, действующая на груз в вагоне, $G=$ $650, \mathrm{\kappa H}$

- сила тяжести, действующая на гружёный вагон, $G_{l}$ $=794, \mathrm{\kappa H}$

- начальная скорость и/или скорость входа вагона в зону затормаживания, $v_{H T 2}=v_{B X T 2}=3.569$, м $/ \mathrm{c}$;

- время затормаживания вагона, вычисленное по формуле (23), $t_{T 2}=1,625 \mathrm{c}$;

- продольный уклон участка второй тормозной позиции, $\sin \psi_{2 T} \approx \psi_{2 T}=0.01$ рад, или $i_{T 2}=10 \%, \cos \psi_{2 T} \approx$ 1 ;

- коэффициент сухого трения скольжения «сталь по стали», $f_{T}=0.25[11]$;

- сила попутного ветра малой величины, $F_{w x}=3.2$ кН;

- сила трения скольжения обода колёсных пар о сжатые тормозные шины, $F_{T O P M}=23.75$ кН [12];

- сила трения скольжения колёсных пар о сжатые тормозные шины (основное сопротивление), $F_{O 2 T}=$ 198.5 кH;

- сила сопротивления от воздушной среды и ветра, $F_{C B}=0.0005 G_{1} \approx 0.4 \mathrm{\kappa H}$

- сила сопротивления от снега и инея, $F_{C H}=$ $0.00025 G_{1} \approx 0.2 \mathrm{\kappa H}$

- приведённая масса вагона с грузом $M_{П Р 0}=$ $8.869 \cdot 10^{4}$, кг

Здесь будем иметь в виду, что для установки тормозных замедлителей на спускной части горки выделены прямые участки, длина которых для второй тормозной позиции, согласно паспорта устройств, применяемых на горке, подбирается при необходимости установки двух замедлителей (например, типа К3-5, НК-14 или ВЗКН). Для устройства второй тормозной позиции может быть предусмотрен прямой участок длиной 25.52 м и зарезервирован участок длиной 10 м для возможного повышения мощности тормозных замедлителей по итогам уточнённых расчётов.

Энергия (см. правую часть формулы (1)), погашаемая двумя замедлителями типа К3-5, должна быть достаточной, чтобы поглотить энергию (см. левую часть формулы (1)), приобретаемую отцепом в процессе скатывания с горки. Например, на второй тормозной по- зиции нечётной сортировочной горки станции «Екатеринбург-Сортировочный» установлены по два замедлителя КЗ-5 мощностью 1.2 м э.в. (метр энергетической высоты) каждый.

\section{4. Результаты}

Для анализа результатов были построены графические зависимости (рис. 1) пути торможения от времени на основе выражений (17), (21) и (22) при изменении $t$ от 1.0 до 2.0 с шагом $\Delta t=0.1 \mathrm{c}$.

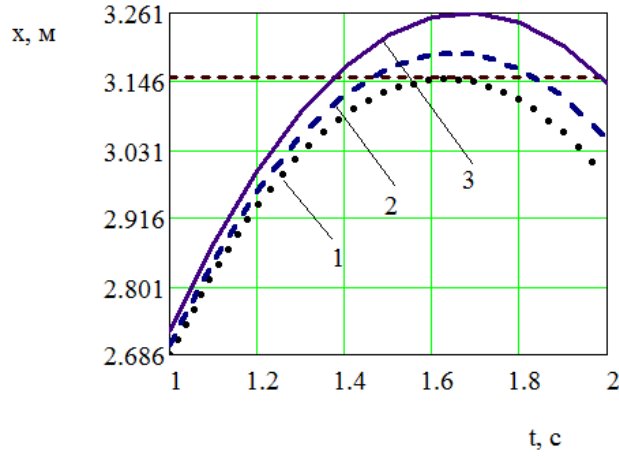

Рис. 1. Зависимости величины тормозного пути от времени торможения, рассчитанные по формулам:

$$
1-(22) ; 2-(17) ; 3-(20)
$$

Из рис. 1 видно, что графики имеют характер возрастающей квадратичной зависимости до момента остановки вагона. Максимальные значения пути торможения $l_{1}=3.152 \mathrm{M}, l_{2}=3.195$ м и $l_{2}=3.262 \mathrm{M}$, что соответствуют времени торможения $t_{1}=1.625 \mathrm{c}, t_{2}=$ 1.648 с и $t_{3}=1.682 \mathrm{c}$.

График зависимости пути торможения от скорости, построенный по результатам расчётов по формуле (9) при изменении $v_{A i}$ от 0 до $5 \mathrm{~m} / \mathrm{c}$ с шагом $\Delta v_{A i}=0.25$ м/с, представлен на рис. 2.

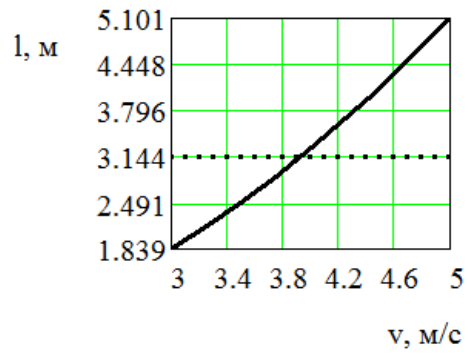

Рис. 2. Зависимость пути торможения от скорости

Как видно из рис. 1 , при $v_{A i}=0$ путь торможения $l_{i}=0$. Это подтверждает рассуждение о важности входа вагона в зону затормаживания тормозных позиций с начальной скоростью $v_{A}>0$. В противном случае произойдёт полная остановка вагона до включения вагонного замедлителя.

При выполнении условия $v_{A}>0$ кинетическая энергия вагона с массой $M$ и начальной скоростью $v_{A}$ будет полностью израсходована на преодоление работы $A_{r}$ силы сопротивления $F_{r}$, появляющейся при включении вагонного замедлителя.

В свою очередь, работа силы сопротивления всякого рода $A_{r}$, накопленная на ободе колеса колёсных 
пар вагона, рельсовых нитей и на тормозных шинах вагонного замедлителя, будет рассеиваться в окружающую среду в виде тепла. При полной остановке вагона, т.е. $v_{B}=0$, будет соблюдено условие $E_{0}+\left(-A_{r}\right)=0$.

\section{5. Обсуждение}

В работе [12] отмечено, что существующая методика расчётов сортировочных горок $[10,13,14]$ в основном направлена на определение высоты сортировочной горки от её вершины до расчётной точки, а такие кинематические параметры движения вагона, как ускорение и время движения вагона в зоне затормаживания, не принимаются во внимание; также в методике отсутствует расчёт длины пути затормаживания вагона.

Результаты расчётов пути торможения вагона с использованием выражения (9) и на основе формулы скорости элементарной физики (11), позволили сделать вывод о том, что при одном и том же значении начальной скорости вагона, результаты расчётов по этим формулам одинаковые.

Обобщая результаты расчётов времени и пути торможения вагона, выполненных с использованием разработанных математических моделей (9), (11) и (17), (21), (22 - 24), можно отметить, что при одном и том же значении начальной скорости, они дают результаты, приемлемые для использования в инженерных расчётах. Это подтверждает корректность и применимость построенных математических моделей применительно к зоне торможения вагона на всех участках тормозных позиций.

Математические модели движения вагона (отцепа) по всей длине участка тормозных позиций сортировочной горки при воздействии попутного ветра малой величины дали возможность разработать новую методику расчёта динамики вагона на этом участке, позволяющую найти кинематические параметры вагона (время и скорость) при заданном геометрическом параметре (длина) рассматриваемого участка сортировочной горки.

Результаты расчётов по определению кинематических параметров вагона по предлагаемой методике позволили по известной величине пройденного расстояния вагона по всей длине участка тормозных позиций («на проход») определить время, в течение которого произойдёт равноускоренное движение вагона на данном участке сортировочной горки.

\section{6. Заключение}

1. Используя теорему теоретической механики об изменении кинетической энергии для несвободной материальной точки в конечной форме, получены формулы расчёта пути торможения вагона в зоне торможения сортировочной горки.

2. Анализ результатов расчётного примера позволил сделать вывод о том, что при одном и том же зна- чении начальной скорости вагона, использование разработанных расчётных формул позволяет получить результаты, приемлемые для выполнения инженерных расчётов. Это подтверждает бесспорность, корректность и применимость построенных математических моделей для определения кинематических параметров вагона применительно к зоне затормаживания вагона на всех участках тормозных позиций сортировочной горки.

3. Результаты выполненных исследований могут быть использованы для корректного решения задач по расчёту и проектированию сортировочных горок.

\section{Список литературы}

1. Kondo O., Yamazaki Y. Simulation Technology for Railway Vehicle Dynamics // Nippon Steel \& Sumitomo Metal Technical Report. 2013, no. 105, pp. 77-83.

2. Негрей В.Я., Пожидаев С.А., Филатов Е.А. Обоснование уровня технического оснащения и оптимизация параметров конструкции сортировочных комплексов железнодорожных станций // Транспортные системы и технологии перевозок. Сборник научных трудов Днипровского национального университета железнодорожного транспорта имени академика В. Лазаряна. 2014, no. 8, pp. 110-119. doi: 10.15802/tstt2014/38098

3. Bardossy M. G. Analysis of Hump Operation at a Railroad Classification Yard // Proceedings of the 5th International Conference on Simulation and Modeling Methodologies, Technologies and Applications. 21.07.2015 23.07.2015, SCITEPRESS - Science and and Technology Publications, pp. 493-500.

4. Boysen N., Emde S., Fliedner M. The basic train makeup problem in shunting yards // OR Spectrum. 2016, vol. 38, no. 1, pp. 207-233. doi: 10.1007/s00291-015-0412-0.

5. Lu C., Shi J. Dynamic response of vehicle and track in long downhill section of high-speed railway under braking condition // Advances in Structural Engineering. 2019, vol. 34, no. 1, 136943321987057. doi: 10.1177/1369433219870573.

6. Polach 0 . Creep forces in simulations of traction vehicles running on adhesion limit // Wear. 2005, vol. 258, 7-8, pp. 992-1000. doi: 10.1016/j.wear.2004.03.046.

7. Dick C. T., Dirnberger J. R. Advancing the Science of Yard Design and Operations With the CSX Hump Yard Simulation System // 2014 Joint Rail Conference. 04022014, American Society of Mechanical Engineers.

8. Бантюкова С. О. Trains breaking-up safety control at hump yards // Eastern-European Journal of Enterprise Technologies. 2015, vol. 3, 3(75), pp. 4-9. doi: 10.15587/1729-4061.2015.42400.

9. Эксплуатационно-технические требования к устройствам сортировоч ных горок. Available at: http://osjd.org/dbmm/download?vp=51\&load=y\&col_id=2066\&id=3185.

10. Козаченко Д. Н., Бобровский В. И., Гревцов С. В., Березовый Н. И. Управление скоростью скатывания отцепов при уменьшении тормозной мощности замедлителей // Наука и прогресс транспорта. Вестник Днепропетровского национального университета железнодорожного транспорта. 2016, vol. 0, 3(63), pp. 28-40. doi: 10.15802/stp2016/74710.

11. Туранов X.T., Гордиенко А.А., Саидивалиев Ш.У. О математическом описании торможения вагона на сортировочной горке // Транспорт: наука, техника, управление (Научный информационный сборник). 2019, no. 7, pp. 27-30.

12. Туранов Х.Т.,Гордиенко А.А., Саидивалиев Ш.У. О подходе к определению некоторых кинематических параметров движения вагона на тормозных позициях сортировочных горок // International Journal of Advanced Studies. 2019, vol. 8, no. 4, p. 122. doi: 10.12731/2227-930X-20184-122-136.

13. Рудановский В.М., Старшов И.П., Кобзев В.А. О попытке критики теоретических положений динамики скатывания вагона по уклону сортировочной горки // Бюллетень транспортной информации. 2016, 6 (252), pp. $20-25$.

14. Пазойский Ю.О., Кобзев В.А., Старшов И.П., Рудановский В.М. К вопросу движения вагона по уклону железнодорожного пути // Бюллетень транспортной информации. 2018, 2 (272), pp. 35-37.

Материал поступил в редакцию 27.10.2019

Туранов Х.Т., Саидивалиев Ш.У. Определение кинематических параметров движения вагона на участках тормозных позиций сортировочной горки // Современные проблемы транспортного комплекса России. 2019. Т.9. №1. С. 21-26 


\title{
THE DETERMINATION OF KINEMATIC PARAMETERS OF THE RAIL CAR'S MOVEMENT ON THE BRAKING POSITIONS OF MARSHALLING YARD
}

\author{
KHabibula Turanov ${ }^{1}$, SHukhrat Saidivaliev ${ }^{2}$ \\ ${ }^{1}$ Ural State University of Railway Transport, Ekaterinburg, Russia \\ ${ }^{2}$ Tashkent Railway Engineering Institute, Tashkent, Uzbekistan
}

Abstract

The existing methods for calculating the marshalling yards are mainly aimed at determining the height of the sorting slide and the kinematic parameters of the movement of the car in the braking zone are not considered. The article presents the calculation formulas and the methodology for determining the braking path of the car in the areas of the brake positions of the marshalling yard. The developed technique is based on a theorem of theoretical mechanics on the change in kinetic energy for a free material point in a final form. It is shown that the path the car passes while going through the breaking positions can be determined by the formula of the path with a known value of acceleration when braking the car. Using the developed formulas, the braking distance was calculated depending on the braking time and the speed of the release. The relative calculation error for the same value of the initial speed did not exceed $3.7 \%$, which confirms the correctness of the developed calculation formulas. The proposed method allows us to determine the time it takes the uniformly accelerated movement of the car in a given section of the sorting slide to take place at a known value of the distance travelled by the rail car in the area of the brake position. The results of the research can be used to correctly solve the problems of calculating and designing of marshalling yards.

Keywords: marshalling yard, braking positions, rail car, kinetic energy, momentum, kinematic parameters, d'Alembert principle, kinetic energy change theorem, theorem on the motion of the center of inertia of a system of material points, theorem on the change in momentum.

\section{References}

1. Kondo O., Yamazaki Y. Simulation Technology for Railway Vehicle Dynamics // Nippon Steel \& Sumitomo Metal Technical Report. 2013, no. 105, pp 77-83.

2. Negrei V.IA., Pozhidaev S.A., Filatov EA. Obosnovanie urovnia tekhnicheskogo osnashcheniia i optimizatsiia parametrov konstruktsii sortirovochnykh kompleksov zheleznodorozhnykh stantsii [Justification of the leve of technical equipment and optimization of design parameters sorting complexes train stations] // Transportnye sistemy i tekhnologii perevozok. Sbornik nauchnykh trudov Dniprovskogo natsionalnogo universiteta zheleznodorozhnogo transporta imeni akademika V. Lazariana [Transport systems and transportation technologies]. 2014, no. 8, pp. 110-119. doi: 10.15802/tstt2014/38098. (In Russ.)

3. Bardossy M. G. Analysis of Hump Operation at a Railroad Classification Yard // Proceedings of the 5th International Conference on Simulation and Modeling Methodologies, Technologies and Applications. 21.07.2015 23.07.2015, SCITEPRESS - Science and and Technology Publications, pp. $493-500$

4. Boysen N., Emde S., Fliedner M. The basic train makeup problem in shunting yards // OR Spectrum. 2016, vol. 38, no. 1, pp. 207-233. doi $10.1007 / \mathrm{s} 00291-015-0412-0$

5 Lu C., Shi J. Dynamic response of vehicle and track in long downhill section of high-speed railway under braking condition // Advances in Structural Engineering. 2019, vol. 34, no. 1, 136943321987057. doi $101177 / 1369433219870573$

6. Polach $\mathrm{O}$. Creep forces in simulations of traction vehicles running on adhesion limit // Wear. 2005, vol. 258, 7-8, pp. 992-1000. doi 10.1016/j.wear.2004.03.046

7 Dick C . T Dirnberger J R Advancing the Science of Yard Design and Operations with the CSX Hump Yard Simulation System // 2014 Joint Rail Conference. 04022014, American Society of Mechanical Engineers.

8. Bantiukova S. O. Upravlinnia bezpekoiu rozformuvannia sostaviv na sortuvalnikh girkakh [rains breaking-up safety control at hump yards] // Vostochno-Evropeiskii zhumal peredovykh tekhnologii [EEJET (Eastern-European Journal of Enterprise Technologies)]. 2015, vol. 3, 3(75), pp. 4-9. doi: 10.15587/1729-4061.2015.42400. (In Ukr.).
9. Ekspluatatsionno-tekhnicheskie trebovaniia $\mathrm{k}$ ustroistvam sortirovochnykh gorok [Operational and technical requirements for marshalling yards]. Available at: http://osjd.org/dbmm/download?vp=51\&load=y\&col id $=2066 \&$ id=3185. (In Russ.).

10. Kozachenko D. M., Bobrovskyi V. I., Grevtsov C. V., Berezovyi M. I. Controlling the speed of rolling cuts in conditions of reduction of brake power of car retarders // Science and Transport Progress. Bulletin of Dnipropetrovsk National University of Railway Transport. 2016, vol. 0, 3(63), pp. 28-40. doi: 10.15802/stp2016/74710

11. Turanov K.T., Gordienko A.A., Saidivaliev S.U. O matematicheskom opisanii tormozheniia vagona na sortirovochnoi gorke [About mathematical description of the car bra king on the marshalling hump] // Transport nauka, tekhnika, upravlenie (Nauchnyi informatsionnyi sbornik) [Transport. science, equipment, management (Scientific Information Collection)]. 2019 no. 7 pp. 27-30. (In Russ.).

12. Turanov K. T., Gordienko A. A., Saidivaliev S. U. O podkhode k opredeleniiu nekotorykh kinematicheskikh parametrov dvizheniia vagona na tormoznykh pozitsiiakh sortirovochnykh gorok [About the approach to the determination of certain kinematic parameters of the car's movement on the hump braking positions of marshalling yards] // Mezhdunarodnyi zhurnal perspektivnykh issledovanii [IJAS (International Journal of Advanced Studies)]. 2019, vol 8, no. 4, p. 122. doi: 10.12731/2227-930X-2018-4-122-136. (In Russ.)

13. Rudanovsky V.M., Starshov I.P., Kobzev V.A. O popytke kritiki teoreticheskikh polozhenii dinamiki skatyvaniia vagona po uklonu sortirovochno gorki [About the attempt of criticism of theoretical positions on dynamics of the car rolling on the marshalling hump slope] // Biulleten transportnoi informatsii [the Bulletin of Transport Information]. 2016, 6 (252), pp. 20-25. (In Russ.)

14. Pazoysky U.O., Kobzev V.A., Starshov I.P., Rudanovsky V.M. K voprosu dvizheniia vagona po uklonu zheleznodorozhnogo puti [On the question of car movement on the railway track incline] // Biulleten transportnoi informatsii [the Bulletin of Transport Information]. 2018, 2 (272), pp. 35-37. (In Russ.).

Received 27/10/2019

Turanov KH.T, Saidivaliev SH.U. The determination of kinematic parameters of the rail car's movement on the braking positions of marshalling yard // Modern Problems of Russian Transport Complex. 2019, vol.9, no.1, pp. 21-26 